Documentation et bibliothèques

\title{
Les dossiers documentaires de la Médiathèque du Musée d'art contemporain de Montréal
}

\section{Régine Francoeur}

Volume 43, numéro 3, juillet-septembre 1997

URI : https://id.erudit.org/iderudit/1033030ar

DOI : https://doi.org/10.7202/1033030ar

Aller au sommaire du numéro

Éditeur(s)

Association pour l'avancement des sciences et des techniques de la

documentation (ASTED)

ISSN

0315-2340 (imprimé)

2291-8949 (numérique)

Découvrir la revue

Citer cet article

Francoeur, R. (1997). Les dossiers documentaires de la Médiathèque du Musée d'art contemporain de Montréal. Documentation et bibliothèques, 43(3),

149-151. https://doi.org/10.7202/1033030ar

Tous droits réservés (C) Association pour l'avancement des sciences et des techniques de la documentation (ASTED), 1997
Ce document est protégé par la loi sur le droit d'auteur. L'utilisation des services d’Érudit (y compris la reproduction) est assujettie à sa politique d'utilisation que vous pouvez consulter en ligne.

https://apropos.erudit.org/fr/usagers/politique-dutilisation/ 


\section{Les dossiers documentaires de la Médiathèque du Musée d'art contemporain de Montréal}

\author{
Régine Francoeur \\ Technicienne en documentation \\ Musée d'art contemporain de Montréal
}

\section{Historique}

Le Musée d'art contemporain de Montréal, créé le 4 juin 1964, entrevoit l'organisation d'une bibliothèque dès 1966 et c'est à partir du $1^{\text {er }}$ septembre 1971 qu'un Centre de documentation, appelé par la suite Médiathèque en raison de la multiplicité des supports de la documentation, est ouvert au public. Le rôle de la Médiathèque consiste à repérer, acquérir, préserver, organiser et transmettre l'information nécessaire à la réalisation des objectifs du Musée d'art contemporain de Montréal. Celui-ci «a pour fonction de faire connaître, de promouvoir et de conserver l'art québécois contemporain et d'assurer une présence de l'art contemporain international et ce, par des acquisitions, expositions et autres activités d'animation.» (Loi sur les musées nationaux; art. 24)

II existe de toute évidence une demande considérable pour une documentation permettant la diffusion de la production artistique. Or, un problème particulier se pose en art contemporain, domaine où les artistes, par définition, produisent depuis peu. «Le Musée d'art contemporain de Montréal choisit de faire débuter l'exercice de son mandat avec la date de la création de la Société d'art contemporain de Montréal en 1939. Le Musée estime en effet que ce moment est important pour l'histoire du pays dans la mesure où il marque l'urgence ressentie par les artistes d'assumer les dynamismes de la création contemporaine et de s'inscrire dans les grands courants internationaux de l'art. » (Musée d'art contemporain de Montréal 1994, 9)

En raison de la rareté des publications portant sur les artistes québécois et canadiens contemporains, le personnel du Musée commence à réunir des documents dits éphémères comme les coupures de journaux qui, avec d'autres, serviront à la création de dossiers documentaires, un type de collection alors méconnu ou peu développé. Ces dossiers «répondent à une demande très forte des consommateurs de l'information dans toutes les formes d'organisation: entreprises, administrations, associations, collectivités, etc.» (Couzinet 1994, 9). «Le dossier permet à l'usager d'obtenir rapidement des réponses qui abordent les divers aspects de la question qu'il se pose. Ce travail de sélection et d'exploitation est donc réalisé pour un utilisateur bien connu, quelle que soit la situation dans laquelle il se trouve: apprentissage, découverte, transmission, décision ou action.» (Couzinet1994, 247)
Au fil des ans, les dossiers se sont étoffés et la demande n'a fait que croître. La Médiathèque possède aujourd'hui une collection de documents dits éphémères classés dans plus de 10500 dossiers (tableau ci-dessous) et $40 \%$ du travail de référence se fait à partir de cette source. En 1971, la biliothèque accueillait 737 chercheurs, 1039 lecteurs et le personnel répondait à 182 demandes par la poste et par téléphone. En 1995, le nombre d'usagers a dépassé 20000 et le personnel de la Médiathèque a répondu à plus de 15 500 questions de référence sur place, par courrier et par téléphone. Durant les deux dernières années, 1110 nouveaux dossiers se sont ajoutés à la collection. Les utilisateurs du site Web, pour le seul mois d'avril 1996, sont au nombre de 1424 .

\begin{tabular}{|c|c|c|c|c|}
\hline \multicolumn{5}{|c|}{$\begin{array}{l}\text { Tableau. ÉVOLUTION DES COLLECTION DE } \\
1971-1996\end{array}$} \\
\hline $1971-1972$ & $1972-1973$ & $1973-1974$ & 1974-1975 & $1975-1976$ \\
\hline 984 & - & - & - & - \\
\hline 1976-1977 & $1977-1978$ & 1978-1979 & 1979-1980 & $1980-1981$ \\
\hline 1950 & 2000 & 2300 & 2393. & 2630 \\
\hline 1981-1982 & 1982-1983 & 1983-1984 & 1984-1985 & 1985-1986 \\
\hline 2700 & - & - & - &. \\
\hline 1986-1987 & $1987-1988$ & 1988-1989 & 1989-1990 & 1990-1991 \\
\hline- & 5000 & 6619 & 7732 & 8728 \\
\hline 1991-1992 & 1992-1993 & 1993-1994 & 1994-1995 & 1995-1996. \\
\hline 8332 & 8818 & 9795 & 10559 & \\
\hline
\end{tabular}




\section{Contenu des dossiers}

La Médiathèque se veut un lieu de recherche sur l'art contemporain québécois, canadien et international. Sa clientèle se compose du personnel du Musée, d'artistes, de chercheurs, professeurs, étudiants, journalistes, collectionneurs, galeristes, muséologues et en général du public intéressé à l'art contemporain. La collection de dossiers documentaires de la Médiathèque constitue donc une source précieuse, un outil indispensable autant pour le créateur qui désire transmettre ses idées, faire part de ses activités que pour l'usager à la recherche d'indices, de pistes, de données sur les réalisations en art contemporain.

Les dossiers contiennent des renseignements d'ordre biographique et bibliographique, sous la forme de curriculum vitæ, de comptes rendus critiques et autres coupures de presse ou de périodiques, de cartons d'invitation, de notes manuscrites, d'affiches, de correspondance et de catalogues. Pour ces derniers, qu'ils soient produits par les galeries, les musées ou les artistes eux-mêmes, la limite maximale de 30 pages a été établie. Ces catalogues, difficiles à trouver en librairie ou dans les bibliothèques publiques, ont en général été produits à l'occasion d'une exposition. Ils peuvent par exemple contenir une liste et des photographies des œuvres exposées, un texte descriptif, critique ou académique, une notice biographique, une bibliographie ou une liste d'expositions.

Arts appliqués, communication, culture, dessin, esthétique, graphisme, gravure, histoire, histoire de l'art, métiers d'art, muséologie, peinture, performance, photographie, sculpture et vidéo constituent les champs de recherche. Les dossiers documentaires se répartissent en six catégories: 1) Biobibliographies d'artistes ou de critiques d'art; 2) Organisations; 3) Événements (biennales, symposiums, concours artistiques); 4) Groupes d'artistes post-1939; 5) Musées; 6) Galeries et centres d'expositions.

\section{Objectifs}

La diffustion des dossiers d'artistes permet aux créateurs de se faire connaître du public. Elle contribue à promouvoir leur travail dans une multitude de champs disciplinaires et à comprendre leur cheminement. Or, les artistes ne sont pas tous représentés par une galerie ou un agent. Pour cette raison, la Médiathèque les invite, eux et leurs associations, galeries, agents ou même collectionneurs, à fournir de l'information pour l'ouverture et la mise à jour de dossiers à leur sujet. Les dossiers documentaires permettent de faire le point sur des sujets d'actualité et de situer les manifestations, les mouvements et les réalisations artistiques. Ainsi, les étudiants en art qui utilisent la collection des dossiers documentaires peuvent enrichir leurs connaissances en général et stimuler leur goût pour l'art en particulier.

En définitive, la diffusion des dossiers et leur mise à jour continue apportent un soutien aux artistes, à ces «créateurs [qui] ajoutent à la valeur d'une société en même temps qu'ils l'expriment et la reflètent. Ce sont eux qui font les sociétés vivantes et dynamiques, en les travaillant comme un ferment, en assurant le triomphe de la vie sur la mort, en liant le passé à l'avenir, en offrant sans cesse à l'homme cette nourriture de l'esprit qui peut seule le combler. C'est pourquoi une société qui se respecte et aspire au respect doit accorder leur juste part aux créateurs.» (Québec (Province) 1980, VI)

\section{Constitution et classement des dos- siers}

En 1971, avant l'irruption de l'informatique, les dossiers sont classés par ordre alphabétique. Petit à petit, la collection de documents éphémères augmente et le classement alphabétique rend la manipulation difficile car le personnel doit à chaque fois décaler les dossiers dans les classeurs lorsqu'il procède à des ajouts. Le fonds documentaire ne cesse d'augmenter, et l'espace se fait de plus en plus rare. Par ailleurs, les projets de déménagement du Musée au centre-ville vont bon train. L'équipe du Centre de documentation (désignation retenue à partir de 1975) opte alors pour un classement par ordre numérique (correspondant à la cote du dossier) de toutes les catégories de dossiers. Le 28 mai 1992, le Musée d'art contemporain de Montréal est inauguré dans son nouvel édifice, sur le quadrilatère de la Place des Arts.
Afin de faciliter la manipulation et la recherche, il s'avère nécessaire de miser sur une bonne conservation et une bonne diffusion des pièces. Pour ce faire, le personnel utilise des pochettes accordéons divisées en trois sections, une première pour les catalogues de moins de trente pages, une deuxième pour les cartons d'invitation et les affiches et une dernière pour les articles de journaux, communiqués, correspondance et lettres manuscrites. Les documents reçus proviennent d'un dépouillement des journaux (effectué par une firme), de conservateurs, de musées et galeries, d'associations, de collectionneurs ainsi que de la collaboration des artistes. II faut un minimum de dix documents ou un catalogue d'exposition pour ouvrir un dossier. Le personnel de la Médiathèque procède à l'indexation et au rangement de la documentation éphémère selon l'ordre alphabétique du descripteur attribué dans l'aire de tri. Le technicien responsable de la création des dossiers documentaires effectue une recherche d'autorité pour tout nouveau descripteur; par ordre de priorité, le personnel de la Médiathèque utilise comme source pour les descripteurs Best Seller, IRIS, ISM, Art Index, les index ou les documents euxmêmes. Une fois l'autorité établie, le technicien procède au catalogage et effectue la saisie dans Best Seller. Un responsable supervise une équipe d'une dizaine de bénévoles qui effectuent, à raison d'une journée par semaine chacun depuis octobre 1988, le classement dans les dossiers ou dans la section «divers». Les nouveaux bénévoles sont soumis à un entraînement de quelques semaines. Un suivi est nécessaire afin de garder une cohérence et une uniformité dans le classement des dossiers facilitant la manipulation et la consultation aux usagers de la Médiathèque. Pour connaître l'existence d'un dossier le bénévole vérifie si le terme d'indexation retenu pour un document apparaît dans la liste alphabétique des descripteurs. Si oui, le document est classé par ordre chronologique dans le dossier (les documents de la dernière section sont perforés et attachés) et la cote du dossier (à six chiffres) ainsi que la date de production du document sont inscrites dans le coin supérieur droit de chacune des pièces. Le dossier renferme une seule copie, sauf pour les cartons d'invitation, affiches et catalogues pour lesquels deux copies sont conservées. En attente de l'ouverture 
du dossier, le bénévole range les documents dans les «divers». Si d'autres pièces se retrouvent dans cette section et que le nombre atteint dix, le technicien responsable procède à l'ouverture du dossier. Un «divers» inactif depuis 15 ans est éliminé.

\section{Autres dossiers}

La collection des dossiers documentaires comprend aussi 1000 dossiers se rapportant aux expositions ainsi qu'aux événements du Musée (ateliers, concerts, conférences, spectacles de danse, débats, films, lectures, performances, rencontres, théâtre). Leur traitement diffère quelque peu de celui des autres dossiers. En effet, des index imprimés sont produits par ordre alphabétique d'événements, par ordre chronologique et par ordre numérique. Un responsable effectue la saisie des événements dans Edibase, logiciel intégré et multilingue de gestion de bases de données textuelles. La documentation pour les dossiers documentaires d'événements publics du Musée d'art contemporain de Montréal, provient du concepteur de l'événement, du ou des artistes participants, du dépouillement des journaux ainsi que des autres services du Musée. Pour effectuer une recherche exhaustive, l'usager doit donc consulter non seulement le dossier de l'artiste, mais aussi les dossiers des événements relatifs à l'artiste.

\section{Consultation}

L'usager peut consulter les dossiers documentaires à partir d'une liste alphabétique. La demande doit se faire au poste de référence, car cette collection est à rayons fermés. Une équipe de techniciens ainsi qu'une bibliothécaire de référence offrent à l'usager une aide à la recherche. L'équipe dirige l'usager afin de lui faciliter la tâche et lui éviter une perte de temps, qu'il s'agisse d'une recherche sur un artiste, un thème ou un mouvement. En plus de lui conseiller livres, périodiques, documents visuels et audiovisuels, le personnel suggère toujours à l'utilisateur la consultation des dossiers documentaires dont la documentation éphémère s'avère unique. La Médiathèque étant un lieu où l'on transmet des connaissances, l'usager doit obtenir l'information la plus complète possible. La Médiathèque utilise la collection des dossiers documentaires comme un outil pédagogique, de recherche et de diffusion du savoir. Les dossiers donnent accès à une multitude d'informations et peuvent aussi bien servir d'amorce à une recherche, compléter un article, étoffer un texte, servir de moyen de découverte ou apporter un éclairage critique. L'usager qui se renseigne sur un objet, par exemple, peut trouver des informations techniques sur cet objet en regard de la production de l'artiste, son contexte, sa valeur. Le goût d'apprendre, l'usager l'a déjà en venant à la Médiathèque. Ce qu'il désire, c'est accroître ses connaissances à partir des outils de recherche offerts à la Médiathèque.

Depuis avril 1996, les usagers d'Internet peuvent accéder au site Web de la Médiathèque du Musée d'art contemporain de Montréal (http:// Media.MACM.qc.ca). L'usager peut y retrouver des informations sur la collection, le personnel et les services offerts ainsi que des bibliographies d'artistes, des bibliographies thématiques spécialisées, des comptes rendus et des textes. La Médiathèque du Musée d'art contemporain de Montréal offre à l'usager plus que des outils généraux sur l'art. Outils de recherche et personnel qualifié pour une recherche de qualité et plus pointue font de la Médiathèque un lieu qui favorise le développement de la mémoire de l'art.

\section{Perspectives}

Toute personne intéressée à l'art contemporain peut en tout temps mettre à jour ou déposer un dossier documentaire à la Médiathèque qui reçoit régulièrement des dossiers provenant d'artistes, de collectionneurs, de galeries, d'agents et d'étudiants. L'artiste qui dépose un dossier offre au public des renseignements sur ses activités et un témoignage sur l'art actuel. Les informations transmises par un artiste permettent de prendre connaissance de sa formation, de son processus de création, de sa place au sein d'une école, d'une tendance, d'une génération, de sa notoriété, de sa position sur le marché de l'art. En procédant à la mise à jour de son dossier, l'artiste contribue à l'avancement des connaissances dans le domaine des arts visuels par la mise en valeur de sa production, de son discours, de ses pratiques artistiques, de sa démarche créatrice et de la profession artistique en général. D'autre part, près de 1000 dossiers sur les événements publics du Musée d'art contemporain de Montréal permettent de brosser un historique du Musée et offrent une vue d'ensemble des artistes présents en art contemporain, connus ou non, qui participent aux expositions collectives ou individuelles, aux conférences, aux débats.

La Médiathèque apporte par la diffusion des dossiers documentaires un soutien aux artistes professionnels et une valorisation à la relève. La Médiathèque témoigne de la présence de l'art contemporain québécois, canadien et international. Elle permet aussi au public de comprendre la contribution sociale de l'artiste et l'importance de l'art dans la société. Enfin, la consultation des dossiers sensibilise le public aux oeuvres d'art, suscite sa curiosité et développe son intérêt pour l'art contemporain. Et c'est avec ardeur que la Médiathèque du Musée d'art contemporain de Montréal, la plus importante bibliothèque spécialisée en art contemporain au Canada, poursuit cette mission depuis plus de vingt-cing ans.

\section{Sources consultées}

Couzinet, Viviane et al. 1994. Le dossier documentaire: conception, réalisation, valorisation. Paris: ADBS Éditions. 268 p.

Jones, Lois Swan. 1990. Art Information: Research methods and resources. $3^{\text {rd }}$ ed. Iowa: Kendall/ Hunt Publishing Company. 373 p.

Jones, Lois Swan and Gibson Sarah Scott. 1986. Art libraries and information services: Development, organization and management. London: Academic Press, Inc. 343 p.

Keaveney, Sydney Starr. 1986. Contemporary art documentation and fine arts libraries. London: Scarecrow Press, Inc. 181 p.

Larsen, John C. 1985. Museum librarianship. Hamden: Library Professionnal Publication. $136 \mathrm{p}$.

Musée d'art contemporain de Montréal. 1994. Politiques du Musée d'art contemporain de Montréal concernant sa collection. Montréal: le Musée. $32 \mathrm{p}$.

Québec (Province). 1980. La juste part des créateurs. Québec: Développement culturel et scientifique. $82 \mathrm{p}$.

Québec (Province). 1984. Esquisse d'une problématique de la condition de l'artiste. Québec: Service gouvernemental de la propriété intellectuelle et du statut de l'artiste. 20 p. 